\title{
¿Qué y cómo observar interacciones para comprender la autoridad pedagógica del profesor en su ejercicio?'
}

Marisa Meza"

Pilar Cox"

Guillermo Zamora"

\section{Resumen}

La autoridad pedagógica del profesor en el aula tal como se ha entendido de manera tradicional en las escuelas ha llegado a su fin. Parece necesario entonces comprender la autoridad que hoy se da en las aulas desde nuevos referentes teóricos y prácticos para formar a los futuros profesores. Una tarea para ello consiste en identificar las formas desde dónde se construye actualmente la autoridad pedagógica del profesor. Este trabajo contribuye a tal tarea presentando los desafíos teórico-prácticos que plantea el proceso de construcción de una propuesta de protocolo de observación de la autoridad pedagógica. Se utiliza una metodología cualitativa que considera tanto el juicio de expertos como una prueba piloto y se asume un modelo de toma de decisiones deliberativo. En relación al qué y cómo observar, la principal conclusión consiste en afirmar que un protocolo de observación sobre autoridad pedagógica que tiene como propósito abrirse a su actual ejercicio debe desplazar la atención desde la figura del profesor a las interacciones. El foco en la interacción permitiría la observación de nuevas formas de legitimación de la autoridad que los jóvenes hoy realizan, permitiendo la superación del sesgo que puedan traer los observadores quienes pertenecen a otra generación.

\section{Palabras clave}

Autoridad pedagógica - Enseñanza secundaria - Observación de clases.

I- Nuestros agradecimientos a la Comisión Nacional de Investigación Científica y Tecnológica de Chile (CONICYT), la que financia el proyecto FONDECYT № 1130863 , dentro del cual se enmarca este trabajo.

II- Pontificia Universidad Católica de Chile, Santiago de Chile, Chile.

Contactos: mmeza@uc.cl; pcox@uc.cl; gzamora@uc.cl 


\title{
What and how to observe interactions to understand teachers' educational authority'
}

Marisa Mezall

Pilar Cox"

Guillermo Zamorall

\begin{abstract}
Teachers' authority in the classroom as traditionally understood in schools has come to an end. Therefore, it seems necessary to understand this authority from a new theoretical and practical perspective to educate future teachers. One of the challenges in this field is to identify the references and approaches from which teachers' educational authority in the classroom is built today. This work contributes to this task by presenting the theoretical and practical challenges involved in building a protocol for observing educational authority. It uses qualitative methodology that includes both the opinion of experts and a pilot application, within a model of deliberative decision-making. In relation to what and how to observe, the main conclusion is that a protocol for observing educational authority that aims at opening to the various ways in which it is exerted in the classroom today should focus not on the teacher but on the interactions in the classroom. This focus on interaction permits observing how today's adolescents legitimize authority, and thus allows overcoming the bias that can be found in observers from a different generation.
\end{abstract}

\section{Keywords}

Teachers' authority - Secondary education - Classroom observation.

I- We acknowledge Comisión Nacional de Investigación Científica y Tecnológica (CONICYT - National Commission for Scientific and Technological Research) which funds the project FONDECYT No. 1,130,863. II- Pontificia Universidad Católica de Chile, Santiago de Chile, Chile.

Contacts: mmeza@uc.cl; pcox@uc.cl; gzamora@uc.cl 


\section{Introducción}

La autoridad pedagógica del profesor en el aula tal como se ha entendido de manera tradicional en las escuelas ha llegado a su fin (ARENDT, 1996; DUBET, 2006; GUILLOT, 2006; MARTUCCELLI, 2009). Diversos estudios socioeducativos plantean las profundas tensiones que atraviesa hoy la relación profesor estudiantes en la educación secundaria. Los estudios evidencian que actualmente los profesores no tienen garantizado ni el respeto, ni la escucha, ni menos el reconocimiento de sus estudiantes (BATALLÁN, 2003; GUILLOTTE, 2003; FLORES; GARCÍA, 2007; GRECO, 2007; OSORIO, 2009; ZAMORA; ZERÓN, 2010; CIDE, 2010).

Parece necesario comprender la actual construcción y ejercicio de la autoridad ampliando nuestros referentes teóricos $\mathrm{y}$ prácticos para formar a los futuros profesores desde nuevas formas de construcción de autoridad. Una tarea consiste en identificar la(s) forma(s) desde dónde se construye y se ejerce hoy la autoridad pedagógica en las aulas. Para ello, es necesario construir instrumentos que nos permitan captar su construcción y ejercicio tal y como se da actualmente. Este trabajo pretende contribuir a tal tarea presentando el proceso de construcción de una propuesta de protocolo de observación de la autoridad pedagógica y los desafíos teórico prácticos que se encuentran al definir qué y cómo observarla en las aulas.

El equipo de investigación se planteó las siguientes interrogantes orientadoras: ¿Cómo construir un marco teórico que permita conceptualizar la autoridad pedagógica sin coartar la captación de posibles nuevos modos de ejercerla y evitando que la conceptualización del fenómeno sea tan laxo que no pueda ser captado? ¿Qué se debe registrar para comprender mejor la o las formas en que los profesores construyen su autoridad en el aula? ¿Cómo entregar directrices para el registro a los observadores sin que éstas sean teñidas por las creencias previas de cada observador sobre la autoridad? ¿Cuál sería la forma más adecuada de validar un protocolo de observación de la autoridad pedagógica en ejercicio? El desarrollo de este trabajo ofrece algunas respuestas a tales interrogantes.

El presente trabajo se desarrolla en cinco partes: la primera, presenta un marco teórico que comprende una aproximación conceptual a la autoridad pedagógica, una caracterización general para su identificación y la dificultad que presentan las creencias implícitas de los observadores junto a una tipología tentativa; la segunda, presenta decisiones clave y una duda en la construcción del protocolo de observación de la autoridad pedagógica; la tercera, presenta el proceso de validación; la cuarta, resultados y sugerencias extraídas del proceso de validación; finalmente, la última parte, discute tanto las decisiones como las posibilidades y dificultades que tensionan la construcción de dispositivos adecuados para la observación de la autoridad pedagógica del profesor en el aula.

\section{Marco teórico}

\section{Aproximaciones al tema de la autoridad pedagógica}

Actualmente existen dos amplias aproximaciones al tema de la autoridad pedagógica: la primera, considera que la autoridad pedagógica está justificada, pero que el ejercicio de su poder no es legítimo y la segunda, considera que la autoridad pedagógica está justificada y es legítima bajo determinadas circunstancias.

La primera aproximación entiende la autoridad pedagógica como un modo de ejercer dominio sobre los alumnos mediante una violencia simbólica. En esta línea se encuentran fundamentalmente los trabajos de Bourdieu y Passeron (1996). La autoridad para ellos sería necesaria para que los profesores ejerzan su acción, en tanto los profesores necesitan ejercer violencia para imponer un arbitrario cultural. Este arbitrario cultural vendría dado desde la institución escolar y, en último término, desde la sociedad, en cuanto ella provee al profesor de 
este poder y sólo puede ejercerlo en cuanto es su representante. Por ello, el maestro sería sólo un imperio dentro de un imperio (BOURDIEU, 1997). Esto significa que la autoridad pedagógica estaría justificada porque surge de una necesidad social, pero no necesariamente sería legítima ante los estudiantes.

A su vez, Kojève (2005) sugiere una forma de violencia simbólica cuando la figura de autoridad apela a la manipulación afectiva, esto es, aludir a la posibilidad de quiebre de un vínculo afectivo directo o indirecto para lograr la obediencia. En este sentido se puede entender estas concepciones de la autoridad en Bourdieu, Passeron y Kojéve como una crítica a la legitimidad y límites de la autoridad pedagógica debido a su concepción acerca del modo arbitrario en que surge y se impone.

La segunda aproximación entiende la autoridad pedagógica como una relación positiva que no sólo está justificada, sino que también es legítima y posee límites éticos. Guillot (2006), por ejemplo, evocando la etimología del término, señala que la autoridad pedagógica hace referencia a la capacidad de autorizar, de hacer crecer y dar vida. La autoridad sería naturalmente constructiva y liberadora, porque daría progresivamente vida al educando para el logro de su autonomía. La legitimidad de la autoridad pedagógica proviene para Guillot de sus fines que no están puestos en la voluntad arbitraria de la institución representada por los profesores sino en la autonomía o bien de los estudiantes.

Por su parte, Bérnger y Pain (1998) definen la autoridad como el poder de hacerse obedecer sin coerción física, a través de una suerte de vínculo tácito entre autorizador y autorizado.

Otras definiciones ponen el énfasis en el consentimiento de la relación de poder (FRANCISCO, 2002), en el liderazgo transformativo (BASS, 1985) o en el reconocimiento mutuo de la jerarquía o asimetría entre las partes (ARENDT, 1996; KOJÈVE, 2005). En otras palabras, todas estas concepciones no sólo justifican la autoridad sino que dan razones para legitimarla mediante los argumentos propios de nuestras sociedades constitucionales democráticas modernas, esto es: el respeto a la autonomía, los derechos de las personas y, el consentimiento mutuo y libre de posibles asimetrías.

Considerando lo anterior y a pesar de enmarcarnos dentro de la segunda aproximación al tema de la autoridad, se decide, en la construcción del instrumento de observación, no excluir la posibilidad de describir el fenómeno de la autoridad desde la perspectiva de la dominación y el ejercicio arbitrario del poder. Si esto surgiera en el proceso de recogida de información.

\section{Caracterización de la autoridad pedagógica y creencias}

Con el fin de caracterizar y distinguir la autoridad pedagógica dentro del mar de interacciones y acontecimientos que suceden entre profesores y estudiantes en las aulas, se presentan a continuación cinco lineamientos extraídos fundamentalmente de los aportes de la teoría de la comunicación de Luhmann (1995; 1998) y el análisis fenomenológico de la autoridad de Kojève (2005) ${ }^{1}$.

\section{a) La autoridad pedagógica es una realidad social}

Luhmann (1995) observa que la autoridad no es un atributo personal, sino un tipo especial de comunicación por el cual se coordinan los sujetos entre sí. La autoridad es una realidad que emerge sólo en la interacción social. Aplicado a la situación del aula, se puede decir que para que se presente la autoridad se requiere de profesores y estudiantes en situación de interacción que construyan una realidad social por medio de comunicaciones. El profesor en soledad no es autoridad. Kojève (2005) agrega a esta perspectiva que la autoridad sólo puede ser reconocida por quien obedece. Lo que enfatiza

1- Tanto en el caso de Luhmann como de Kojève se tematiza directamente la autoridad y eso dará luces para distinguir la autoridad pedagógica en su ejercicio. 
la idea que la autoridad no es un atributo de la persona, sino un reconocimiento que pueden hacer unos de otros en interacciones concretas.

\section{b) La autoridad pedagógica consiste en la posibilidad de actuar sobre otro}

La autoridad se presenta cuando la comunicación de un sujeto (el profesor) sirve de premisa para las decisiones de otros sujetos (estudiantes), los cuáles han tenido abierta la posibilidad de negar tal comunicación. El que tiene autoridad da razones para la acción. De este modo, una persona ejerce autoridad en la medida que logra que el otro acepte sus demandas y renuncie a otras posibilidades de actuación que podrían ser igualmente atractivas. Al respecto, Kojève (2005) advierte que la autoridad presupone la posibilidad de una oposición y la renuncia consciente y voluntaria a la realización de esa posibilidad. En este sentido la autoridad es la posibilidad que tiene un agente de actuar sobre los demás (o sobre otro) y que estos renuncien a otras posibilidades de acción, teniendo la posibilidad de hacerlo.

c) La autoridad pedagógica consiste en un intercambio de demandas y aceptaciones

La autoridad pedagógica se manifiesta particularmente en los intercambios de solicitudes de parte del profesor y aceptaciones de parte de los alumnos. Las solicitudes contienen, a su vez, una información - lo que se espera que el otro haga - y una expresión la forma, el tono, en que se estructura aquella información -. El modo de expresión influye en la probabilidad de que el otro atienda y acepte la petición. Por otra parte, las aceptaciones contienen principalmente una comprensión y una interpretación de lo que está en juego. En tal sentido, la autoridad pedagógica aparece cuando el profesor logra que el alumno atienda, comprenda y acepte sus demandas ya sean estas una orden, una prohibición o un permiso y por ello, la autoridad requiere reconocimiento, que se expresa en las acciones de aceptación. Utilizando los términos de Arendt (1996), se puede señalar que la autoridad siempre demanda una obediencia aceptada.

\section{d) La autoridad pedagógica es frágil}

Las relaciones sociales - comunicativas - son frágiles. Permanentemente existe la posibilidad de que un sujeto no atienda, ni entienda las expresiones de su interlocutor. El rechazo es una posibilidad latente en cualquier interacción social. Luhmann (1998) plantea la contingencia de las partes en la problemática de las relaciones sociales. El concepto de contingencia refiere a que un sistema o una persona siempre puede decidir de otro modo o dar un no por respuesta. Esto significa que la autoridad no es una condición social permanente o definitiva, más bien, en cada momento está la posibilidad de una contra reacción. Kojève (2005) refuerza la idea indicando que la autoridad solo lo es, cuando es reconocida por quien la acepta y perdura mientras es reconocida y aceptada. No reconocer una autoridad es negarla, destruirla. Pero esto no significa que la autoridad se agote en eventos sin historia. El intercambio de demandas y aceptaciones constituiría un momento de una trama mayor, porque en la interacción hay tanto un movimiento previo como uno posterior que abre o cierra posibilidades de acción futuras (LUHMANN, 1995; 1997). Es por ello, que se debe hablar de un proceso de construcción de autoridad más que un acto de autoridad (LUHMANN, 1997). La autoridad entendida como fenómeno se evidenciaría en actos de autoridad, pero se trata de actos que van formando una trama, una historia con actos previos y posteriores que determinan (nunca causalmente), los que vendrán en el futuro. En el caso de la relación profesor-estudiante, el profesor no puede fijar en términos absolutos las posibilidades de decisión de sus estudiantes, pero puede 
ir construyendo una relación que haga más posible la aceptación de sus demandas futuras.

\section{e) La autoridad pedagógica es una realidad activa}

Se ha dicho anteriormente que la autoridad aparece en los intercambios en que hay solicitudes desde una parte, las cuales son atendidas y aceptadas por la contraparte, a pesar de que ésta tenga otras posibilidades de acción. De acuerdo con esta definición, se puede decir que no hay relación de autoridad cuando no hay demandas ni peticiones. La autoridad requiere de una intervención, ella es activa, no pasiva. Al respecto Kojève (2005) señala incluso que si los otros igualmente van a actuar, independiente de la petición, prohibición o permiso, entonces la autoridad no se manifiesta. En otras palabras, para que haya autoridad pedagógica se requiere que sea el profesor el que dé lugar a las acciones de sus estudiantes (o a la inhibición de ellas), introduciendo nuevas razones para la acción como lo indica Raz (1990), logrando que hagan algo que no harían espontáneamente (o dejen de hacer algo que harían espontáneamente).

A partir de los planteamientos teóricos analizados, se definieron los siguientes cursos de acción para el proceso de observación de aula: dado que la autoridad es un fenómeno relacional y social, ésta se observará a partir del momento en que comienzan las interacciones entre profesores y estudiantes; la autoridad consiste en la posibilidad de actuar sobre otro por lo que será foco de atención cuando el profesor realiza una demanda; la autoridad consiste en un intercambio de demandas y aceptaciones, por ello se observará cuando el profesor ordena, prohíbe o permite que se realice una acción y los estudiantes aceptan tales demandas, prohibiciones o permisos teniendo otras posibilidades de acción; la autoridad es una realidad frágil, debido a lo cual se observará más de una vez las interacciones entre profesores y estudiantes y en el momento en que se construye la relación de autoridad, esto es, antes de que se establezcan rutinas de relación en el aula, pues aunque la autoridad puede negarse en cualquier momento, se construye un cierto patrón de comportamiento al comenzar las interacciones (COREY, M; COREY, G, 1997; CANESSA, 1999); la autoridad es activa, esto significa que se debe prestar atención a los eventos en la clase en que los estudiantes hacen o dejan de hacer algo que sin la intervención del profesor no harían o dejarían de hacer.

\section{Tipos de autoridad}

La aceptación de las demandas (mandatos, prohibiciones, permisos), lo que es al mismo tiempo, la renuncia a otros cursos de acción, se debe a diversos motivos. Esas motivaciones dan lugar a la determinación de diversos tipos de autoridad. En otras palabras, los tipos de autoridad responden a la pregunta: ¿por qué acepto la autoridad de tal agente sobre mí? ¿Qué razones se dan y logran la aceptación o seguimiento de las demandas que se realiza a los estudiantes? Se identifican las siguientes razones para lograr la aceptación: el primer tipo de razones se relaciona con la experticia, con el saber especializado al que se apela al realizar la demanda o dar una respuesta. Esta se denomina autoridad del experto o sabio (RAZ, 1990; KOJÈVE, 2005; ZAMORA; ZERÓN, 2010; MEZA, 2011). El segundo tipo de razones se relaciona con la capacidad de dirimir conflictos o establecer relaciones de justicia mediante el establecimiento de reglas de convivencia en el aula. Se trata de la autoridad justa (RAZ, 1990; KOJĖVE, 2005; ZAMORA; ZERÓN, 2010). El tercer grupo de razones, con la capacidad de proponer nuevas ideas o cursos de acción. Se trata de la autoridad del líder. El cuarto tipo de razones se relaciona con la vinculación a la tradición, la institución educativa y los logros realizados en el pasado. Se trata de la autoridad de la tradición. El quinto se vincula con la preocupación, cuidado y respeto por los estudiantes y por la apelación a razones morales para motivar la aceptación. Se denomina autoridad moral. El sexto grupo de razones se 
relaciona con el vínculo con los estudiantes y el compromiso afectivo que eso genera. Se trata de la autoridad amoroso-manipuladora (KOJÈVE, 2005; ZAMORA; ZERÓN, 2010; MEZA, 2011).

Considerando que la finalidad del protocolo de observación consiste en identificar las formas de construcción y ejercicio de la autoridad pedagógica del profesor en el aula, que la autoridad pedagógica puede ser comprendida de diversos modos (justificada, legítima o ilegítima), que puede ser reconocida (aceptada, obedecida) debido a un diverso grupo de razones y que las investigaciones actuales sobre creencias epistemológicas y teorías implícitas en sus diversos enfoques establecen un vínculo entre las concepciones personales con los modos en que los individuos perciben hechos y acontecimientos que llevan a los individuos a predecir acciones propias y ajenas (HOFER; PINTRICH, 1997; PECHARROMÁN; POZO, 2010; LÓPEZ-VARGAS; BASTOTORRADO, 2010; GÓMEZ; GUERRA, 2012), nos enfrentamos al siguiente desafío: por una parte, un protocolo de observación debe garantizar que las observaciones que se realicen levanten información pertinente respecto al fenómeno a observar, por lo que parece necesario que el protocolo incluya instrucciones y ciertas informaciones para los observadores que lo utilizarán, pero por otra parte, las instrucciones e información que se entreguen deben evitar que los observadores que utilicen el instrumento sólo registren aquellos eventos de autoridad pedagógica que ellos conceptualicen de acuerdo a su personal visión de lo que ella signifique.

\section{Decisiones y una duda en la construcción de protocolo de registro de la autoridad pedagógica}

Considerando las reflexiones teóricas sobre autoridad pedagógica, sus consecuencias prácticas, se decide que el protocolo de observación debe:
1. Permitir recoger información comparable a partir de los registros de diversos observadores. Con este fin el protocolo incluye la solicitud de informaciones acerca del número de observaciones, fechas en que se realiza, duración de las observaciones, procedimientos a seguir antes y después de la observación, datos de lo que las actividades antes y después de la clase, así como, la verificación de condiciones que permitan un registro unificado.

2. Dirigir la observación a clases en que se haya reconocido a un(a) profesor(a) como autoridad. Debido a ello, se observarán las clases de profesores que hayan sido identificados por sus estudiantes como autoridad pedagógica mediante un cuestionario previo.

3. Permitir la observación de momentos de construcción y ejercicio de la autoridad, asumiendo que ello se da en un contexto en donde existen actos previos y posteriores que forman una trama. Por ello se decide observar las primeras clases de los profesores pues en ellas parece ser más evidente el modo en que se establecerán como autoridad en un nuevo período académico y se solicitan informaciones respecto al número de clases observadas.

4. Centrar la atención en la autoridad pedagógica entendida como una realidad social, que se da en una interacción. Por ello, el protocolo indica que se comienza a registrar una vez que se producen interacciones entre profesores y estudiantes en el aula y no se registra lo que profesores o estudiantes realizan por separado sino las demandas y aceptaciones, los modos en que interactúan unos con otros.

La duda que permaneció tiene que ver con el grado de amplitud o focalización que debía poseer el protocolo en los registros de aula considerando la diversidad de creencias acerca de su significado que traerían inevitablemente los usuarios del protocolo de observación. Por un lado, se consideraba recomendable identificar y comunicar claramente a los observadores las diferentes formas que podía tomar la construcción y ejercicio de la autoridad pedagógica y buscar el registro específico de ese tipo de interacciones 
mediante definiciones generales o entrega de ejemplos. Focalizar si la observación involucraba capacitar a los observadores en la identificación de diversos modos de ejercicio de la autoridad pedagógica, según la mirada teórica del estudio. Esta estrategia tiene la ventaja de favorecer que los observadores tengan elementos para identificar las interacciones más relevantes para el estudio y así asegurar en mayor medida su registro detallado y la desventaja de cerrar la posibilidad de registro de nuevas formar de construcción y ejercicio de la autoridad pedagógica no previstos en el marco teórico.

Por otra parte, se evaluó la pertinencia de un registro amplio, de todas las interacciones de aula, para luego, en el proceso de codificación y análisis, levantar las categorías que fuesen necesarias, manteniendo ajeno al observador de aspectos específicos del fenómeno. Esta estrategia suponía concentrar la capacitación en el registro amplio de observación sin entrenar en el reconocimiento de situaciones específicas. Esta estrategia tiene la ventaja de no sesgar la observación a partir de concepciones teóricas previas y de las creencias propias de cada observador sobre lo que es o no es autoridad pedagógica y cómo esta se presenta en el aula. La fundamental ventaja de esta alternativa consiste en la apertura a lo nuevo, que es parte constitutiva del objetivo de investigación, pues justamente se quiere comprender nuevas formar de construcción y ejercicio de la autoridad pedagógica en las aulas, pero la posible desventaja de que los registros más interesantes de captación de lo nuevo fuesen superficiales debido al intento de los observadores de registrar todas las interacciones existentes entre profesores y estudiantes. Por ello, esta duda se constituyó en uno de los focos centrales del proceso de validación.

\section{Proceso de validación}

El proceso de validación del protocolo de observación se realizó en vista a asegurar que las decisiones tomadas fuesen las adecuadas en relación a los objetivos de la investigación tanto en relación con las informaciones que se entregaban como con las solicitudes que se hacían. Por ello, se decide realizar un proceso de validación en dos fases (HERNÁNDEZ; FERNÁNDEZ; BAPTISTA, 2010): la primera, mediante juicio de expertos y la segunda, mediante la realización de una prueba piloto.

La validación del contenido del protocolo mediante juicio de expertos (HERNÁNDEZ; FERNÁNDEZ; BAPTISTA, 2010) se realizó mediante la técnica de pensamiento en voz alta o think aloud (HIMMEL; INFANTE, 2005). Se seleccionó a tres especialistas para este proceso. El criterio de selección fue su experticia de al menos cinco años en observación de aula con fines de investigación o de evaluación docente. Cada miembro del equipo investigador se reunió con uno de los expertos al que se le presentaron los objetivos y el protocolo de observación en versión impresa. Luego, se solicitó que lo leyera en voz alta frente al investigador y que verbalizara sus dificultades de comprensión o de lectura, sus sugerencias y sus observaciones en base a su conocimiento y experiencia, en vistas a la optimización de la efectividad de la observación de acuerdo a los objetivos y los tópicos de decisiones clave, a saber: si la información e indicaciones que se entregaban eran adecuadas y si la tarea solicitada era suficiente para recoger información pertinente al objetivo. Posteriormente, se estructuró una tabla comparativa de los comentarios de los tres expertos organizándolos por categoría con el fin de hacerlos comparables y facilitar el establecimiento de aspectos comunes y diferentes entre ellos. El equipo discutió y realizó modificaciones al protocolo para evaluarlo en una prueba piloto.

La prueba piloto del protocolo de observación se realizó intentando replicar lo más posible la instancia de observación real. Se seleccionó, en un mismo establecimiento particular subvencionado, ${ }^{2}$ a tres profesores

2- Esto significa que se trata de un colegio con administración y dirección privada que recibe subvención del estado chileno. En Chile, este tipo de colegios representan el $60 \%$ del total de colegios existentes en educación media (SIMCE, 2012). 
identificados por la directora de la institución como aquellos a los que los alumnos más obedecen. Se observó a un profesor de lenguaje y comunicación, una profesora de inglés y una profesora de matemática. A cada profesor se le observó en 4 ocasiones con cursos diferentes pertenecientes todos a la enseñanza secundaria, con un número de alumnos de alrededor de 37 por curso. Con estas medidas se perseguía aumentar la posibilidad de que se tratara de interacciones en que efectivamente fuese posible observar el fenómeno de la autoridad pedagógica, en diferentes formas introduciendo diferencias de asignatura que enseñan los observados y diferencias de género, dentro de un tipo común de colegio en Chile.

Una limitación la constituyó el que la observación piloto se realizara en el segundo semestre del año, ya que si bien se logró observar interacciones, no se trató de aquellas que la construyen inicialmente, sino de las propias de una etapa de trabajo de un grupo, con normas y modos de relación ya consolidados (COREY, M; COREY, G, 1997). Se consideró, a pesar de ello, adecuado porque se trataba de validar el contenido del protocolo y su utilidad para el registro y no de evaluar el modo en que se codificarían esos datos, es decir, el qué y el cómo del ejercicio y no el comienzo del proceso.

\section{Resultados}

De ambos procesos de validación se levantaron recomendaciones que se sistematizan a continuación. Estas recomendaciones se consideraron siguiendo el criterio de significatividad en relación a los objetivos de la observación y las preguntas para la validación.

En relación a la primera pregunta planteada para la validación, referida a la amplitud o focalización de los registros de aula, se generaron las siguientes recomendaciones:

- Privilegiar registro amplio, ya que al observador puede resultarle complejo atender a focos, interpretando y clasificando lo que observa, al tiempo de registrar todo lo que sucede. Se confirma la instrucción: registren todas las interacciones.

- Incorporar aspectos prácticos del registro relacionados con la información relevante para el estudio, como la codificación de quién inicia la intervención, género de los que intervienen, especial énfasis en el registro del tiempo y en el registro de respuestas verbales y no verbales.

- Incorporar referentes a etapas de la clase, como inicio, desarrollo o cierre, para contextualizar las interacciones.

Revisar la redacción del protocolo para evitar excesivo foco en el profesor y no en las interacciones entre profesores y estudiantes en donde existan respuestas verbales y no verbales de aceptación o rechazo. En relación a la segunda pregunta, respecto de la cantidad de información sobre el fenómeno a observar que se debía entregar a los observadores, las sugerencias apuntaron principalmente a:

- Dar instrucciones de observación amplia, sin enfatizar en los focos o formas posibles de autoridad, con el fin de propiciar una observación no sesgada.

- Incorporar una sección interpretativa final, en que los observadores registren aquellos aspectos que más sobresalientemente relacionen con interacciones que reflejen autoridad pedagógica y que los justifiquen con evidencias extraídas de las observaciones. Realizar una pequeña capacitación a los observadores para centrar el registro en las interacciones entre profesor y estudiantes.

Finalmente, se realizaron las siguientes sugerencias en torno a aspectos organizativos, técnicos y de formato:

- Realizar una reunión previa con el profesor a observar para acordar aspectos prácticos y aclarar dudas de sentido.

- Establecer un texto de presentación patrón destinado al observador en el aula para evitar entregar información que pudiese perturbar la observación de la clase.

- Preparar un formato de breve comentario del observador al profesor al finalizar cada clase observada. 
- Incorporar en el protocolo recomendaciones prácticas sobre el manejo de los aspectos de respaldo tecnológico del proceso y que resguarden el normal desarrollo de la clase observada.

- Estructurar el protocolo de manera simple, que permita un registro amplio, sin columnas.

Elaborar el plano de la clase en hoja completa aparte para mantenerlo a la vista durante la observación haciendo referencia a estudiantes que intervienen con consideración de género.

\section{Discusión y decisiones finales}

El modelo de discusión y de toma de decisiones finales se inspiró en un enfoque deliberativo (WALDRON, 2004), esto es, más que la frecuencia de las recomendaciones recibidas, fue relevante en las decisiones las razones que se dieron para fundamentarlas en vistas al logro de los objetivos de observación. A partir de este proceso, el equipo investigador acordó las siguientes decisiones finales: en relación a la amplitud o focalización del registro de observación, se decide optar por un modo amplio, que busca registrar todas las interacciones de la clase observada, del profesor con uno, varios o todos los estudiantes, iniciadas tanto por éste como por uno o más estudiantes. Se descartó con ello también la observación centrada en hitos, entendidos como interacciones clave de la clase, debido a la dificultad de juzgar al comienzo de una interacción, si esta será o no crucial. El registro amplio permite identificar interacciones clave en la fase de análisis, esto es, a posteriori. Esta decisión obliga a tener en cuenta que la premura del registro de todas las interacciones puede hacer perder de vista algunas que sean cruciales para el análisis posterior, por esa razón se decide y recomienda registrar en audio y video cada una de las clases observadas.

Se agrega, una vez finalizado el proceso de registro, una pregunta a los observadores que consulta acerca de las principales estrategias que utilizó el profesor observado para lograr la aceptación de demandas y lineamientos por parte de sus estudiantes y una pregunta de opción múltiple en que se pide determinar si el profesor se parece más a: un juez o una jueza, un dictador o una dictadora, un experto o experta, un amigo o amiga, un guía espiritual, pastor o sacerdote, un líder y también se pide que dé ejemplos de respaldo de esa percepción. Las categorías de definición de estilo son levantadas a partir de la teoría e incluyen la categoría Otro con el fin de evidenciar la posibilidad de registro de otros modos de ejercicio de la autoridad.

Esta solución al problema de la amplitud o focalización para el registro de las observaciones posee diversas ventajas. Posee la ventaja de distinguir entre dos momentos en el trabajo de los observadores: el de registro descriptivo de las interacciones que se realiza durante la observación y el de juicio general y justificación de este juicio a partir de la descripción realizada. El registro amplio también posee la ventaja de permitir obtener datos que no correspondan a la construcción y ejercicio de la autoridad pedagógica dados por el marco teórico o, las creencias previas de los observadores acerca de lo que la autoridad pedagógica sea. El momento del juicio y justificación permite obtener juicios de los observadores susceptibles de ser contrastados a través de los registros obtenidos, pues deben incluirse evidencias obtenidas de los registros y también permite informar implícitamente a los observadores acerca de la existencia de diversos modos de ejercicio y construcción de la autoridad pedagógica en el aula.

La principal limitación consiste en no poder evitar que los observadores intenten replicar su primer juicio a la segunda $\mathrm{y}$ tercera observación. En este sentido y pese a los esfuerzos para evitarlo, los investigadores deben contar con que los observadores posean diversas creencias acerca de lo que sea la autoridad pedagógica, incluso aquellas que la consideran injustificada y/o ilegítima $\mathrm{y}$ 
que estas creencias se puedan de algún modo deslizar a través de los registros. El registro en audio y video son clave a la hora de despejar dudas como la descrita.

En relación al grado de conocimiento a entregar a los observadores sobre el fenómeno conceptual en estudio, se decide centrar la capacitación de los observadores en el registro descriptivo de las observaciones de modo de evitar sesgos. Se indicará solamente el objetivo del estudio que se realiza. Se enfatizará que se está observando a un(a) profesor(a) que es identificado(a) por sus estudiantes como el/ la que más obedecen y que nos interesa saber qué sucede en su clase que puede originar esta opinión por parte de los estudiantes y que la observación se centra en registrar todas las interacciones verbales y no verbales ocurridas en el aula cada cinco minutos.

Esta decisión posee la ventaja de entregar la información justa, intentando evitar posibles sesgos y concentrar la capacitación en el modo en cómo obtener información relevante para la codificación, análisis e interpretación de los datos posteriores. Una posible limitación es causar cierto desconcierto en los observadores y la posible pérdida de interacciones relevantes debido a la presión de registrarlas todas.

En relación a la estructura del protocolo de observación, se decide afinar la información entregada previa y posteriormente al trabajo de registro. Esta instrucción se orienta a asegurar información comparable a partir de registros de diversos observadores. En vista a este objetivo, el protocolo de observación se estructura finalmente en tres secciones. La primera sección presenta indicaciones generales como: 1) orientaciones generales sobre la realización del proceso; 2) indicaciones sobre cómo coordinar la observación con la institución y el profesor a observar y qué resguardar el día de la observación en torno a aspectos prácticos y técnicos a considerar para el proceso; 3) orientaciones específicas para el registro escrito de la observación, indicando códigos específicos y poniendo foco en dos aspectos: por una parte la necesidad de registrar todas las interacciones, entre el profesor (a) y los (as) estudiantes y por otra, considerar que los profesores a observar fueron seleccionados por ser identificados por sus estudiantes como con autoridad pedagógica o a los que tus compañeros más le obedecen, a través de un cuestionario aplicado previamente y que interesa saber por qué se ha generado esa opinión en los estudiantes.

La segunda sección establece un formato para el registro que incluye datos generales del curso y contexto a observar (institución, asignatura, profesor, curso, fecha de observación, hora de inicio y término número de estudiantes, lugar que ocupa la clase de esos estudiantes con ese profesor dentro del año lectivo, distribución por género). Se solicita también realizar una breve descripción del espacio físico, la actividad previa y posterior del curso observado y el tema u objetivo de la clase. Esto porque el protocolo de observación debe evidenciar la idea de que la autoridad es un proceso constructivo, constituido por actos previos y posteriores que van formando una trama y que ocurre en un contexto. Se utiliza el siguiente formato:

\section{Protocolo fase introductoria}

Cuadro 1- Hoja de registro de observación de clases autoridad pedagógica: información general

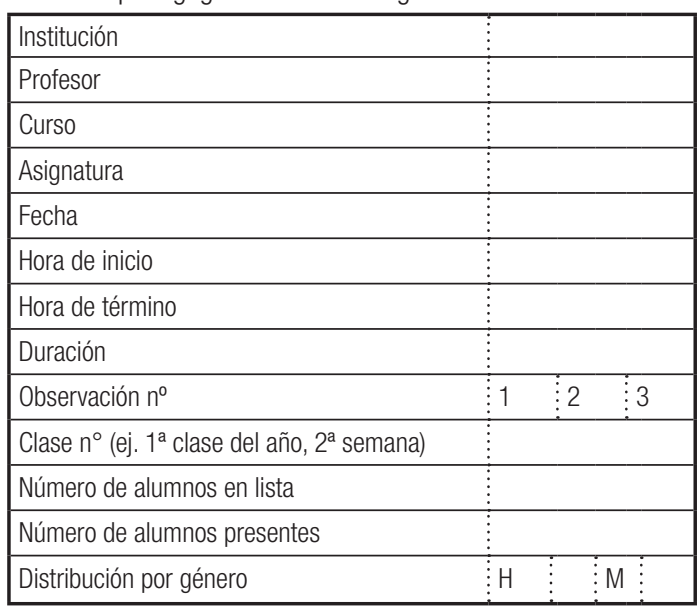

Fuente: elaboración de los autores. 
Cuadro 2 - Hoja de registro de observación de clases autoridad pedagógica: descripción del contexto

\begin{tabular}{|l|}
\hline $\begin{array}{l}\text { Breve descripción del espacio físico. Dibujar un plano sencillo de la } \\
\text { organización de la sala, poniendo número a los alumnos para tenerlo } \\
\text { a la vista e identificarlos en las interacciones. }\end{array}$ \\
\hline $\begin{array}{l}\text { Datos sobre actividad previa del curso (ej. vienen de ed. Física / } \\
\text { vienen de una prueba). }\end{array}$ \\
\hline \\
\hline Tema u objetivo de la clase. \\
\hline
\end{tabular}

Fuente: elaboración de los autores.

Luego, el protocolo contiene un espacio para el registro en el que se solicita registrar las interacciones identificando hora (se recomienda marcar al menos cada cinco minutos para referencia) y actor que inicia la interacción (profesor o estudiantes). El registro de estos datos permitirá levantar información que permita dialogar con la idea de que la autoridad requiere de intervención, esto es, que la autoridad se caracteriza por ser activa y no pasiva.

La tercera sección del protocolo, se orienta a la fase pos observación de interacciones. En ella, se solicita caracterizar las estrategias del profesor para lograr la aceptación y seguimiento de sus demandas a los estudiantes y se pide caracterizar al profesor dentro de un estilo solicitando evidencias tomadas de los registros, como en el formato que se observa a continuación:

\section{Fase de cierre}

Cuadro 3 - Apreciación del observador

Desde su perspectiva, ¿cuáles son las estrategias principales 0 más características que el(la) profesor(a) utilizó para lograr que los alumnos aceptaran sus demandas o lineamientos durante la clase?

Fuente: elaboración de los autores
Cuadro 4 - Tipos de relación de autoridad más relevantes durante la observación: se parece más a...

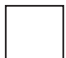

Un juez o una jueza

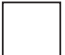

Un dictador 0 una dictadora

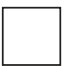

Un experto o una experta

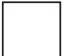

Un amigo o una amiga

Un guía espiritual, pastor o sacerdote

Un(a) líder

De algunos ejemplos que expliquen su caracterización

Fuente: elaboración de los autores.

En este trabajo se ha narrado el proceso involucrado en la creación de un protocolo de observación para acceder a posibles nuevas formas de construcción y ejercicio de la autoridad pedagógica en las aulas. El mayor desafío en este proceso puede resumirse en la tensión que se genera al intentar captar nuevos aspectos o formas de un fenómeno sabiendo, por una parte, que los que realizarán las observaciones - y los propios investigadores poseen fuertes creencias y conceptualizaciones del fenómeno y sabiendo, por otra parte, que se necesitan marcos conceptuales previos para poder reconocer el fenómeno como tal. Desfocalizar la atención desde la figura del profesor a la interacción entre profesores y estudiantes permitirá, a nuestro juicio, superar estas tensiones y la percepción de lo nuevo. 


\section{Referencias}

ARENDT, Hannah. Entre el pasado y el futuro. Barcelona: Península, 1996.

BASS, Bernard. Leadership and performance beyond expectations. New York: The Free Press, 1985.

BATALLÁN, Graciela. El poder y la autoridad en la escuela. La conflictividad de las relaciones escolares desde la perspectiva de los docentes de infancia. Revista Mexicana de Investigación Educativa, México DF., v. 8, n. 19, p. 679-704, sep./dic. 2003.

BÉRNGER, Peter; PAIN, Jean. L'autorité et l'école: fin de système. Ville École Intégration, Paris, n. 112, p. 134-161, mars 1998.

BOURDIEU, Pierre. Capital cultural, escuela y espacio social. Madrid: Siglo XXI, 1997.

BOURDIEU, Pierre; PASSERON, Jean-Claude. La reproducción: elementos para una teoría del sistema de enseñanza. Ciudad de México: Laia, 1996.

CANESSA, Luis. Manual de tecogrupos. Santiago de Chile: Pontificia Universidad de Chile, 1999. 130 p. Documento de estudio - Facultad de Educación, Pontificia Universidad de Chile, Santiago de Chile, 1999.

CIDE - Centro de Investigación y desarrollo de la Educación; ENCUESTA A ACTORES DEL SISTEMA EDUCATIVO 2010, 8., 2010. VIII encuesta a actores del sistema educativo 2010. Santiago de Chile: CIDE/Universidad Alberto Hurtado, 2010.

COREY, Marianne; COREY, Gerald. Groups: process and practice. California: Brooks/Cole, 1997.

DUBET, François. El declive de la institución. Barcelona: Gedisa, 2006.

FLORES, Luis; GARCÍA, Mauricio. Figuras estructurales de la violencia escolar: hacia una recuperación de la subjetividad educativa. Santiago de Chile: Proyecto FONDECYT, Fondo Nacional de Ciencia y Tecnología, 2007.

FRANCISCO, María de Fátima Simões. Autorité et contrat pédagogique chez Rousseau. La Géme biennale de l'education et de la formation, Paris, Juillet, 2002. Disponible en: <http://www.inrp.fr/biennale/6biennale/Contrib/affich. php?\&mode=long\&NUM=377>. Acceso en: 20 dic. 2013.

GÓMEZ, Viviana; GUERRA, Paula. Teorías implícitas respecto a la enseñanza y el eprendizaje: ¿Existen diferencias entre profesores en ejercicio y estudiantes de pedagogía? Estudios Pedagógicos, Valdivia, v. 38, n. 1, p. 25-43, 2012.

GRECO, María Beatriz. La autoridad (pedagógica) en cuestión: una crítica al concepto de autoridad en tiempos de transformación. Rosario: Homo Sapiens, 2007.

GUILLOT, Gerard. La autoridad en la educación: salir de la crisis. Madrid: Popular, 2006.

GUILLOTTE, Alain. Violencia y educación: incidentes, incivilidades y autoridad en el contexto escolar. Buenos Aires: Amorrortu, 2003.

HERNÁNDEZ, Roberto; FERNÁNDEZ, Carlos; BAPTISTA, Pilar. Metodología de la investigación. México, DC: McGraw Hill, 2010.

HIMMEL, Erika; INFANTE, Marta. Evaluación formativa de preguntas de prueba mediante la metodología de la entrevista de pensamiento en voz alta. Boletín de Investigación Educacional, Santiago, v. 20, n. 1, p. 33-49, 2005.

HOFER, Bárbara; PINTRICH, Paul. The development of epistemological theories: beliefs about knowledge and knowing and their relation to learning. Review of Educational Research, Berkeley, v. 67, n. 1, p. 88-140, 1997.

KOJĖVE, Alexander. La noción de autoridad. Madrid: Nueva Visión, 2005.

LÓPEZ-VARGAS, Brenda; BASTO-TORRADO, Sandra. Desde las teorías implícitas a la docencia como práctica reflexiva. Revista Educación, Bogotá, v. 13, n. 2, p. 275-291, mayo/ago. 2010. 
LUHMANN, Niklas. Poder. Ciudad de México: Universidad Iberoamericana, 1995.

LUHMANN, Niklas. Teoría de Ios sistemas sociales: artículos. Ciudad de México: Universidad Iberoamericana, 1998.

MARTUCCELLI, Danilo. La autoridad en las salas de clase. Problemas estructurales y márgenes de acción. Diversia, Valparaíso, n. 1, p. 99-128, abr. 2009.

MEZA, Marisa. La autoridad pedagógica en contexto democrático. Santiago de Chile: Universidad de Chile, 2011. 203 p. Tesis (Doctorado) - Facultad de Filosofía y Humanidades, Universidad de Chile, Santiago de Chile, 2011.

OSORIO, Fernando. Ejercer la autoridad: un problema de padres y maestros. Buenos Aires: Noveduc, 2009.

PECHARROMÁN, Isisdro; POZO, José Ignacio. ¿Cómo sé que es bueno? Creencias epistemológicas en el dominio moral. Revista de Educación, Madrid, v. 353, p. 387- 414, sep./dic. 2010.

RAZ, Joseph. Authority. New York: New York University Press, 1990.

SIMCE. Informe nacional de resultados SIMCE. Santiago de Chile: Ministerio de Educación, 2012.

WALDRON, James. Deliberación, desacuerdo y votación. In: HONGJU KOH, Harold; SLYE, Ronald C. (Ed.). Democracia deliberativa y derechos humanos. Barcelona: Gedisa, 2004. p. 249-267.

ZAMORA, Guillermo; ZERÓN, Ana María. Caracterización y sentido actual de la autoridad pedagógica en escuelas chilenas de sectores de pobreza. Revista Española de Pedagogía, Madrid, v. 68, n. 245, p. 99-116, ene./abr. 2010.

Recibido en: 29.01.2014

Aprobado en: 13.08.2014

Marisa Meza es doctora en filosofía. Académica del Departamento de Teoría y Política de la Educación, Facultad de Educación, Pontificia Universidad Católica de Chile.

Pilar Cox es magíster en educación. Académica Departamento de Psicopedagogía del Aprendizaje, Facultad de Educación, Pontificia Universidad Católica de Chile.

Guillermo Zamora es doctor en educación. Académico Departamento de Teoría y Política de la Educación, Facultad de Educación, Pontificia Universidad Católica de Chile. 